\title{
A non-cytotoxic regimen of decitabine to treat refractory T-cell large granular lymphocytic leukemia
}

\author{
Misam Zawit $^{1}$ | Carmelo Gurnari ${ }^{1,2}$ | Simona Pagliuca ${ }^{1,3}$ | Hassan Awada ${ }^{1}$ | \\ Jaroslaw P. Maciejewski ${ }^{1}$ | Yogen Saunthararajah ${ }^{1}{ }^{\infty}$ \\ ${ }^{1}$ Translational Hematology and Oncology Research Department, Taussig Cancer Center, Cleveland Clinic, Cleveland, OH, USA \\ ${ }^{2}$ Department of Biomedicine and Prevention \& Ph.D in Immunology, Molecular Medicine and Applied Biotechnology, University of Rome Tor Vergata, \\ Rome, Italy \\ ${ }^{3}$ Université de Paris, Paris, France
}

\author{
Correspondence \\ Yogen Saunthararajah, Department of \\ Translational Hematology and Oncology \\ Research, Lerner Research Institute, \\ Cleveland Clinic, 9620 Carnegie Ave \\ $\mathrm{N}$ building, NE6-250, Cleveland, $\mathrm{OH}$ \\ 44106, USA. \\ Email: saunthy@ccf.org \\ Funding information \\ Edward P. Evans Foundation; National \\ Cancer Institute, Grant/Award Number: \\ P30 CA043703 and RO1 CA204373; \\ American-Italian Cancer Foundation; \\ National Heart, Lung, and Blood Institute, \\ Grant/Award Number: PO1 HL146372, \\ R01HL118281, R01HL123904, \\ R01HL132071 and R35HL135795; \\ Henry and Marilyn Taub Foundation
}

\begin{abstract}
We report on a novel, successful, non-cytotoxic therapy to treat multiply-refractory T-LGL in an elderly patient.
\end{abstract}

\section{K E Y W O R D S}

decitabine, frail patients, non-cytotoxic regimen, T-cell large granular lymphocytic leukemia

\section{1 | BACKGROUND}

T-cell large granular lymphocytic leukemia (T-LGL) is characterized by clonal expansion of cytotoxic $\mathrm{T}$ cells and cytopenias of one or more myeloid lineages. We describe a case of T-LGL with red cell transfusion dependence refractory to multiple lines of established therapies, but successfully treated with decitabine administered by a subcutaneous regimen designed to target the epigenetic regulator DNA methyltransferase 1 (DNMT1) without cytotoxicity, a first report of a novel non-cytotoxic molecular-targeted therapy for this disease.
T-cell large granular lymphocyte leukemia (T-LGL) is a chronic lymphoproliferative disorder characterized by clonal expansion of cytotoxic T cells and cytopenia of one or more myeloid lineages. ${ }^{1}$ Despite a typically indolent natural history, most patients eventually require treatment because of severe cytopenias, caused by either direct (cellular-mediated) or indirect (cytokines) attack of myeloid precursors by the clonal T cells. ${ }^{1,2}$ Because T-LGL in its severe form is a rare condition, no head-to-head comparisons of different treatment options have been conducted. Guided by single-arm studies, three drugs-methotrexate (MTX), cyclosporine (CsA), and

Misam Zawit and Carmelo Gurnari contributed equally.

This is an open access article under the terms of the Creative Commons Attribution-NonCommercial-NoDerivs License, which permits use and distribution in any medium, provided the original work is properly cited, the use is non-commercial and no modifications or adaptations are made.

(C) 2021 The Authors. Clinical Case Reports published by John Wiley \& Sons Ltd. 
cyclophosphamide $(\mathrm{Cy}$ - are routinely used as first-line therapies. Overall response rates (ORR) have been reported at $38 \%-60 \%$ for MTX, $45 \%-92 \%$ for CsA, and $47 \%-72 \%$ for $\mathrm{Cy},{ }^{2,3}$ and a recent retrospective analysis reported comparable ORR of $61.5 \%-74.4 \%$ for all three agents. ${ }^{4}$ Even less data exist as to the management of refractory cases. Several drugs inhibiting epigenetic repressing enzymes of the histone deacetylase (HDAC) class are approved for treating other peripheral T-cell malignancies, and a case report described the successful use of the HDAC-inhibitor belinostat $(1000 \mathrm{mg} /$ $\mathrm{m}^{2}$ intravenously, days $1-5$ in 21-day cycles) to produce red cell transfusion independence in a patient with T-LGL and anemia refractory to MTX, CsA, and Cy. ${ }^{5}$ HDAC-inhibitors, however, cause cytopenias in their own right, via cytotoxic or cytostatic actions possibly inherent to HDAC inhibition, which can undermine the cytopenia-correction goal of care. We describe here an elderly patient with T-LGL with red cell transfusion-dependent refractory to several lines of therapy including an HDAC-inhibitor, who was successfully treated with decitabine administered by a regimen designed to inhibit/deplete the epigenetic repressing enzyme DNA methyltransferase 1 (DNMT1) without cytotoxicity. ${ }^{6-9}$

\section{CASE REPORT}

A 79-year-old man with coronary artery disease, chronic kidney disease (CKD) stage III, and non-melanocytic skin cancer presented to his primary care physician in June 2019 with shortness of breath and decreased exercise tolerance. Initial work-up revealed macrocytic anemia (hemoglobin [Hb] $9.4 \mathrm{~g} / \mathrm{L} ; \mathrm{MCV} 105 \mathrm{fL}$ ), absolute neutrophil count of $1.6 \times 10^{9} / \mathrm{L}$, and normal platelet count $\left(180 \times 10^{9} / \mathrm{L}\right)$. Abnormal large granular lymphocytes (LGL) were noted on blood smear and represented $80 \%$ of total circulating lymphocytes (absolute lymphocyte count $1.5 \times 10^{9} / \mathrm{L}$ ). A bone marrow (BM) evaluation showed moderate erythroid hyperplasia, normal granulopoiesis and megakaryopoiesis, no dysplastic changes or chromosomal abnormalities, and infiltration by abnormal cytotoxic $\mathrm{T}$ cells confirmed by flow cytometry (Figure 1A). Molecular studies (nextgeneration sequencing panel of 62 recurrently mutated genes in hematological malignancies) revealed a canonical STAT3 $^{\mathrm{Y} 640 \mathrm{~F}}$ mutation (variant allelic frequency 11.5\%), and T-cell receptor PCR showed a clonal T-cell rearrangement, confirming the diagnosis of T-LGL ${ }^{1,2}$ (recurrent mutations linked with myeloid malignancies were not detected). The anemia was progressive, resulting in red cell transfusiondependence and prompting initiation of therapy with MTX $15 \mathrm{mg}$ weekly. During 6 weeks of MTX therapy, fatigue worsened and red cell transfusion needs increased, leading to MTX discontinuation and the initiation of 2nd-line therapy with prednisone $60 \mathrm{mg}$ daily for 3 weeks with subsequent tapering, for a total duration of 8 weeks. Again, no response was observed, prompting initiation of 3rd-line therapy with CsA $2 \mathrm{mg} / \mathrm{kg}$ daily in divided doses along with short course of prednisone (40 mg daily tapered over 14 days). Over 4 months of CsA therapy, there was no decrease in red cell transfusion requirements, and kidney toxicity required CsA dose-reduction. At this point, the patient was referred to our center and a BM evaluation was repeated: Morphological observations were similar to initial presentation-expanded erythropoiesis and T-LGL cell infiltration. Two cytogenetic abnormalities, not observed at initial presentation, were detected ( $-\mathrm{Y}$ in $3 / 20$, and +8 in $4 / 20$ metaphases). Clonal hematopoiesis with $-\mathrm{Y}$ is not uncommon in older individuals, and trisomy 8 has been reported in association with T-LGL, ${ }^{10,11}$ but since there were no dysplastic changes in the myeloid lineages the diagnosis remained unchanged. Nephrotoxicity (increase in serum creatinine level by $~ 30 \%$ over pre-treatment baseline) prompted dose-reduction in CsA, and together with continued lack-of-response then prompted a switch to 4th-line therapy with tacrolimus (4 $\mathrm{mg}$ daily in divided doses). Also, at this time it was noted that the patient's erythropoietin level was inappropriately low in the context of anemia $(90 \mathrm{mU} / \mathrm{ml})$; thus, epoetin alpha $(60,000$ units biweekly) was incorporated into therapy (as per indication of anemia caused by CKD). Red cell transfusion needs did not decrease after 12 weeks of this treatment, prompting the addition of MTX $10 \mathrm{mg}$ weekly to the tacrolimus/epoietin combination. After 12 weeks of tacrolimus, MTX, and epoetin therapy, there was no decrease in transfusion needs; therefore, the tacrolimus and MTX were discontinued, and the HDAC-inhibitor vorinostat $400 \mathrm{mg}$ per os daily), approved for the treatment of other peripheral T-cell malignancies, was initiated as 5th-line therapy. Over a 3 month period, this treatment was complicated by anorexia, diarrhea, and worsening of fatigue, without decrease in red cell transfusion requirements. Vorinostat was thus discontinued, and the patient was initiated on 6th-line therapy: a non-cytotoxic, DNMT1-targeting regimen of subcutaneous decitabine $0.2 \mathrm{mg} / \mathrm{kg}$ weekly. Red cell transfusion independence was achieved $\sim 7$ weeks after initiation of this therapy, accompanied by subjective improvements in the patient's symptoms and sense of well-being, and with no side effects reported (Figure 1B).

\section{3 | DISCUSSION}

MTX has been proposed to have a specific role for treating STAT3-mutated T-LGL, which in general appears to have worse survival outcomes than wild-type counterparts ${ }^{12,13}$; nevertheless, there is no consensus as to the optimal treatment algorithm for T-LGL, whether STAT3-mutated or otherwise. ${ }^{1}$ A non-cytotoxic molecular-targeted therapy would 

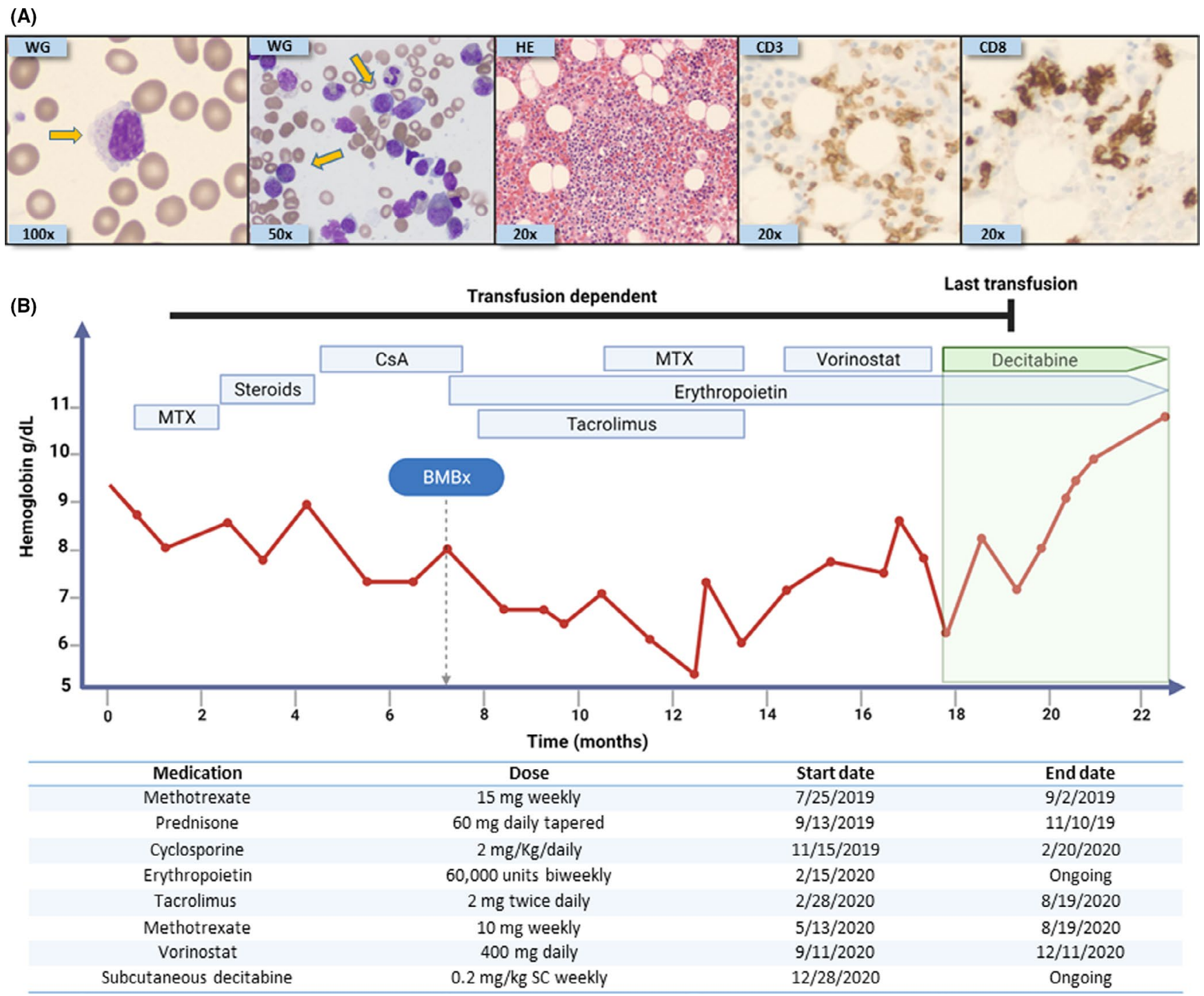

F I G U RE 1 Histopathological findings and treatment course of our index case. (A) From left to right: Wright-Giemsa (WG) stain of peripheral blood showing a classical large granular lymphocyte (100X); WG stain of bone marrow (BM) aspiration showing classical LGL (50X); hematoxylin-eosin stain (20X) of the BM biopsy core with infiltration of small lymphocytes characterized by CD3 (20X) and CD8 (20X) positivity by immunostaining. Flow cytometry confirmed the aberrant T-LGL phenotype: CD2, CD3, CD5 dim, CD8, CD16, and CD57. Yellow arrows indicate large granular lymphocytes. (B) Timeline depicting the type of treatment received along with duration of administration, with dosages described underneath. MTX: Methotrexate, CsA: Cyclosporine, BMBx: Bone marrow biopsy, and SC: subcutaneously

be ideal, since the major goal of therapy is to improve low blood counts, a goal that can be undermined by cytotoxicity, especially in elderly patients who have reserves of functional hematopoiesis already diminished by age and disease processes. As a molecular target, DNMT1 has been (i) clinically validated for treating other T-cell malignancies; (ii) pre-clinically validated for the treatment of T-LGL ${ }^{14}$; and (iii) we had previously developed and described a dose, route-of-administration and schedule of decitabine to deplete DNMT1 without cytotoxicity to BM. ${ }^{6-9}$ The specific non-cytotoxic, DNMT1-targeting mechanism-of-action/ pharmacodynamic effect of this regimen has been documented previously in patients with myeloid malignancies and $\beta$-hemoglobinopathies ${ }^{6-9}$ and represented the rationale for its use even after the failure of another epigenetic modulator, vorinostat, for which separation of cytotoxic from epigenetic pharmacodynamic effects has not been demonstrated. These data and experience taken altogether prompted our first use of this decitabine regimen to treat an elderly patient with T-LGL and red cell transfusion dependence refractory to multiple lines of more established therapies. The subjective and objective clinical response, safety profile, and clinical responses reported for other T-cell malignancies treated with DNMT1-targeting drugs, suggest that non-cytotoxic DNMT1-targeting should be further evaluated to treat T-LGL. 


\section{ACKNOWLEDGMENTS}

We thank Edward P. Evans Foundation, the HENRY \& MARILYN TAUB FOUNDATION, and grants to JPM: R01HL118281, R01HL123904, R01HL132071, and R35HL135795; the American-Italian Cancer Foundation PostDoctoral Research Fellowship (to CG); grants to YS: National Heart, Lung and Blood Institute PO1 HL146372, National Cancer Institute P30 CA043703, National Cancer Institute RO1 CA204373. Published with written consent of the patient.

\section{CONFLICT OF INTEREST}

YS has equity in EpiDestiny. There is no financial and research support. YS received intellectual property-patents around tetrahydrouridine, decitabine, and 5-azacytidine (US 9,259,469 B2; US 9,265,785 B2; US 9,895,391 B2) and cancer differentiation therapy (US 9,926,316 B2).

\section{AUTHOR CONTRIBUTIONS}

M.Z., C.G., and Y.S. wrote the manuscript. S.P., H.A., and J.P.M kindly reviewed the clinical data and helped edited the manuscript. All authors participated in critical review of the final paper and approved the submitted version.

\section{ETHICAL STATEMENT}

This study was approved by the Local Ethics Committee (institutional review board-IRB-approval number 5024). The patient signed an institutional informed consent in which he expressed his will to allow the use of medical record and laboratory data and the publication of this information for research purposes. All procedures were carried out in accordance with guidelines set forth by the Declaration of Helsinki.

\section{DATA AVAILABILITY STATEMENT}

All data will be available upon request to the corresponding author.

\section{ORCID}

Yogen Saunthararajah (10 https://orcid. org/0000-0002-9757-1031

\section{REFERENCES}

1. Lamy T, Moignet A, Loughran TP Jr. LGL leukemia: from pathogenesis to treatment. Blood. 2017;129(9):1082-1094.

2. Sanikommu SR, Clemente MJ, Chomczynski P, et al. Clinical features and treatment outcomes in large granular lymphocytic leukemia (LGLL). Leuk Lymphoma. 2018;59(2):416-422.
3. Moignet A, Lamy T. Latest advances in the diagnosis and treatment of large granular lymphocytic leukemia. Am Soc Clin Oncol Educ Book. 2018;38:616-625.

4. Dong N, Castillo Tokumori F, Isenalumhe L, et al. Large granular lymphocytic leukemia - a retrospective study of 319 cases. Am J Hematol. 2021;96(7):772-780.

5. Poh C, Arora M, Ghuman S, Tuscano J. Belinostat in relapsed/ refractory T-cell large granular lymphocyte leukemia. Acta Haematol. 2021;144(1):95-99.

6. Awada H, Mahfouz RZ, Kishtagari A, et al. Extended experience with a non-cytotoxic DNMT1-targeting regimen of decitabine to treat myeloid malignancies. Br J Haematol. 2020;188(6):924-929.

7. Saunthararajah Y. Key clinical observations after 5-azacytidine and decitabine treatment of myelodysplastic syndromes suggest practical solutions for better outcomes. Hematology Am Soc Hematol Educ Program. 2013;2013:511-521.

8. Saunthararajah Y, Hillery CA, Lavelle D, et al. Effects of 5-aza-2'-deoxycytidine on fetal hemoglobin levels, red cell adhesion, and hematopoietic differentiation in patients with sickle cell disease. Blood. 2003;102(12):3865-3870.

9. Saunthararajah Y, Sekeres M, Advani A, et al. Evaluation of noncytotoxic DNMT1-depleting therapy in patients with myelodysplastic syndromes. J Clin Invest. 2015;125(3):1043-1055.

10. Loughran TP Jr, Kadin ME, Starkebaum G, et al. Leukemia of large granular lymphocytes: association with clonal chromosomal abnormalities and autoimmune neutropenia, thrombocytopenia, and hemolytic anemia. Ann Intern Med. 1985;102(2):169-175.

11. Durrani J, Awada H, Kishtagari A, et al. Large granular lymphocytic leukemia coexists with myeloid clones and myelodysplastic syndrome. Leukemia. 2020;34(3):957-962.

12. Shi M, He R, Feldman AL, et al. STAT3 mutation and its clinical and histopathologic correlation in T-cell large granular lymphocytic leukemia. Hum Pathol. 2018;73:74-81.

13. Barilà G, Teramo A, Calabretto G, et al. Stat3 mutations impact on overall survival in large granular lymphocyte leukemia: a single-center experience of 205 patients. Leukemia. 2020;34(4):1116-1124.

14. Teramo A, Gattazzo C, Passeri F, et al. Intrinsic and extrinsic mechanisms contribute to maintain the JAK/STAT pathway aberrantly activated in T-type large granular lymphocyte leukemia. Blood. 2013;121(19):pp. 3843-3854, S3841.

How to cite this article: Zawit M, Gurnari C, Pagliuca S, Awada H, Maciejewski JP, Saunthararajah $\mathrm{Y}$. A non-cytotoxic regimen of decitabine to treat refractory T-cell large granular lymphocytic leukemia. Clin Case Rep. 2021;9:e04533. https://doi. org/10.1002/ccr3.4533 